\title{
Membrane Cholesterol in Skeletal Muscle: A Novel Player in Excitation-Contraction Coupling and Insulin Resistance
}

\author{
G. Barrientos, ${ }^{1,2}$ P. Sánchez-Aguilera, ${ }^{1,3}$ E. Jaimovich, ${ }^{1,4}$ C. Hidalgo, ${ }^{1,2,5}$ and P. Llanos ${ }^{1,3}$ \\ ${ }^{1}$ Center for Molecular Studies of the Cell, Facultad de Medicina, Universidad de Chile, Santiago, Chile \\ ${ }^{2}$ Physiology and Biophysics Program, ICBM, Facultad de Medicina, Universidad de Chile, Santiago, Chile \\ ${ }^{3}$ Institute for Research in Dental Sciences, Facultad de Odontología, Universidad de Chile, Santiago, Chile \\ ${ }^{4}$ Cell and Molecular Biology Program, ICBM, Facultad de Medicina, Universidad de Chile, Santiago, Chile \\ ${ }^{5}$ BNI, Facultad de Medicina, Universidad de Chile, Santiago, Chile
}

Correspondence should be addressed to P. Llanos; pllanos@odontologia.uchile.cl

Received 5 December 2016; Accepted 6 February 2017; Published 6 March 2017

Academic Editor: Bernard Portha

Copyright (c) 2017 G. Barrientos et al. This is an open access article distributed under the Creative Commons Attribution License, which permits unrestricted use, distribution, and reproduction in any medium, provided the original work is properly cited.

\begin{abstract}
Membrane cholesterol is critical for signaling processes in a variety of tissues. We will address here current evidence supporting an emerging role of cholesterol on excitation-contraction coupling and glucose transport in skeletal muscle. We have centered our review on the transverse tubule system, a complex network of narrow plasma membrane invaginations that propagate membrane depolarization into the fiber interior and allow nutrient delivery into the fibers. We will discuss current evidence showing that transverse tubule membranes have remarkably high cholesterol levels and we will address how modifications of cholesterol content influence excitation-contraction coupling. In addition, we will discuss how membrane cholesterol levels affect glucose transport by modulating the insertion into the membrane of the main insulin-sensitive glucose transporter GLUT4. Finally, we will address how the increased membrane cholesterol levels displayed by obese animals, which also present insulin resistance, affect these two particular skeletal muscle functions.
\end{abstract}

\section{Introduction}

The physiological relevance of plasma membrane cholesterol levels has attracted increased attention in recent years. Cholesterol is an essential component of eukaryotic membranes, which display molar ratios of cholesterol to phospholipids in the range of 7-55 mol\% [1-4]. Physiological levels of cholesterol in the cellular membranes are critical to preserve membrane fluidity and thickness and to structure the lipid domains that are involved in signal transduction processes $[2,5]$.

Contraction of skeletal muscle takes place via the excitation-contraction (EC) coupling process [6]. Action potential propagation into the fiber interior through the transverse tubule (T-tubule) system initiates EC coupling, which results in the cytoplasmic $\mathrm{Ca}^{2+}$ increase that triggers muscle contraction [7]. In addition to contraction, insulin-sensitive glucose transport and glucose homeostasis represent additional key functions of skeletal muscle which occur predominantly at the level of the T-tubule system [8]. Accordingly, T-tubule composition and structure are likely to regulate both the EC process and insulin-sensitive glucose transport.

The mammalian T-tubule membranes are highly enriched in sphingomyelin and cholesterol compared to the surface sarcolemma [9]. This feature endows these membranes with a highly ordered lipid environment [10]. We have reported recently that single fibers isolated from adult skeletal muscle display a $26 \%$ decrease in cholesterol content following incubation with the cholesterol removing agent methyl- $\beta$ cyclodextrin $(\mathrm{M} \beta \mathrm{CD})$. This agent also alters the distribution pattern of the voltage-dependent calcium channel Cavl.1 in T-tubules and suppresses electrically evoked $\mathrm{Ca}^{2+}$ transients [11].

Skeletal muscle is the largest body organ in nonobese subjects and represents the major site of insulin-stimulated glucose disposal [12]. Insulin increases glucose uptake into skeletal muscle and adipose tissue by redistributing type 4 glucose transporters (GLUT4) from their intracellular 
location to the plasma membrane [13]. In skeletal muscle, insulin resistance (IR) is associated with disturbed insulin signaling, leading to defective GLUT4 traffic to the T-tubules and the surface membrane [8].

Animals fed a high-fat diet (HFD) become insulinresistant and exhibit elevated levels of membrane cholesterol compared with normal chow-fed animals [14]. Likewise, mice fed a HFD become obese, develop IR, and display increased levels of cholesterol in isolated skeletal muscle T-tubule membranes and adult muscle fibers [15]. Conversely, new insights into GLUT4 trafficking reveal that compounds that partially reduce membrane cholesterol content increase insulinindependent GLUT4 translocation and glucose uptake, both in adipocytes [16] and in muscle cell lines [14]. Therefore, altering the physiological levels of membrane cholesterol may lead to cellular malfunction and thus may contribute to the pathological processes triggered in humans by obesity or by cholesterol depletion caused by pharmacological agents. Here, we will review the critical evidence that supports a role of membrane cholesterol as a new player in physiological muscle function and in the IR condition.

\section{Role of Cholesterol in Plasma Membrane Properties}

Cholesterol is the single most abundant lipid molecule of plasma membranes, representing up to $55 \mathrm{~mol} \%$ of the total lipid composition [1-4]. The cholesterol molecule is essential for membrane biogenesis [4] and influences the structure and physical properties of biological membranes, including membrane thickness [17] and fluidity [18]. Membrane cholesterol participates in a wide range of physiological functions including limiting ion leakage through membranes [19], modulation of signal transduction pathways [5], and traffic of membrane proteins [20].

Cholesterol is a polycyclic amphipathic molecule derived from a sterane backbone (Figure 1). It has a polar head formed by a single hydroxyl group which in membranes can form hydrogen bonds with polar groups of proteins or lipids [21]. The nonpolar section of cholesterol has two faces, a planar face called the $\alpha$-surface and a rough face called the $\beta$-surface. These sections allow specific cholesterol interactions with protein $\alpha$-helical regions and $\beta$-surfaces $[21,22]$.

Cholesterol modifies the organization of lipids in artificial bilayers. Phosphatidylcholine molecules with unsaturated hydrocarbon chains can adopt a liquid-disordered fluid phase in bilayers; however, cholesterol addition induces a change to the liquid-ordered phase, decreasing the fluidity of the membrane [23-25]. The spatial distribution of cholesterol responds according to the umbrella model, whereby the polar head groups of phospholipid function as an umbrella, shielding the hydrophobic moiety of cholesterol molecules from water [26]. Cholesterol has higher affinity for sphingolipids, leading to a highly regular distribution in membranes which minimizes cholesterol-cholesterol contact [26].

Lipid rafts are small-scale $(10-200 \mathrm{~nm})$ domains found in live cell membranes enriched in cholesterol and sphingolipids. In lipids rafts, the interaction of cholesterol with sphingolipids is more stable; cholesterol presents its $\alpha$-surface to these lipids leaving its $\beta$-surface to interact with transmembrane domains of integral proteins [21]. Lipid rafts play an essential role in membrane-protein sorting and in the formation of signaling complexes [27-29]. In these lipids domains, cholesterol increases the order of lipid-acyl chains, increasing the local membrane thickness and limiting the type of integral membrane proteins located on this hydrophobic environment. These changes around membrane proteins can modulate the local lipid environment and modify the internal protein conformation states and their function [30].

A previous report [31] described a Cholesterol Recognition/Interaction Amino Acid Consensus sequence (CRAC domain), which is a short linear amino acidic motif with a specific vectorial direction (from $\mathrm{N}$ - to C-terminal). The CRAC domain starts at the N-terminus with a Leu (L) or Val (V) residue, followed by a segment comprising 1 to 5 residues. The segment continues with a mandatory Tyr (Y) residue, a segment comprising 1 to 5 residues, and ends with a basic Lys $(\mathrm{K})$ or $\operatorname{Arg}(\mathrm{R})$ residue, $(\mathrm{L} / \mathrm{V})-\mathrm{X}_{1-5^{-}}(\mathrm{Y})-\mathrm{X}_{1-5^{-}}$ $(\mathrm{K} / \mathrm{R})$ [32]. CRAC domains interact with cholesterol in the cytoplasmic leaflet of the membrane. These motifs belong to some transmembrane protein domains and have a favorable fit for cholesterol binding [21].

Another newly recognized cholesterol-binding sequence, known as CARC domain, has recently been described; it has almost the same sequence as CRAC but runs in the opposite direction (from $\mathrm{C}$ - to $\mathrm{N}$-terminal) and has a central aromatic amino acid, which can be either Tyr or Phe, (K/R)- $\mathrm{X}_{1-5^{-}}$ $(\mathrm{Y} / \mathrm{F})-\mathrm{X}_{1-5}-(\mathrm{L} / \mathrm{V})$ [33]. In both cases, the van der Waals forces and $\mathrm{H}$-bonds between the $\mathrm{Y}$ residue and the $\mathrm{OH}$ group of the cholesterol molecule participate in the interaction between cholesterol and the CRAC/CARC-containing protein [21].

Both CRAC and CARC motifs represent oriented amino acid sequences, with an apolar amino acid residue at one terminal and a highly polar, positively charged basic residue at the other end [32]. In some cases, these amino acidic sequences are located in the same transmembrane segment but CARC is associated with the outer leaflet and CRAC is located at the inner leaflet of the plasma membrane. There are several examples of membrane receptors that have such a dual interaction with CRAC and CARC. These receptors include neuropeptide FF receptor, metabotropic glutamate receptor 5, GABA type B receptor subunit 2, CB1 receptor, 5-HT7 receptor, adenosine receptor $\mathrm{A} 1$, VIP receptor 1 , prolactinreleasing peptide receptor, oxytocin receptor, TRVP1 receptor, and corticotrophin-releasing factor receptor 1 [34].

The alignment of the dipolar components of phospholipids at the water interface generates a membrane-internal potential known as the dipole potential [35], with an estimated magnitude around $280 \mathrm{mV}$ [36]. It has been suggested that the dipole potential modulates the translocation rates of ions across lipid membranes [37]. The activities of $\mathrm{Na}^{+} / \mathrm{K}^{+}$ATPase and phospholipase A2 increase with increasing dipole potential $[38,39]$. Cholesterol intercalation strongly affects the dipole potential; cholesterol removal generates a reduction close to $50 \mathrm{mV}$ [40]. Restoration of cholesterol levels reverses this reduction in natural membranes [41]. However, cholesterol levels have negligible effects on the dipole 


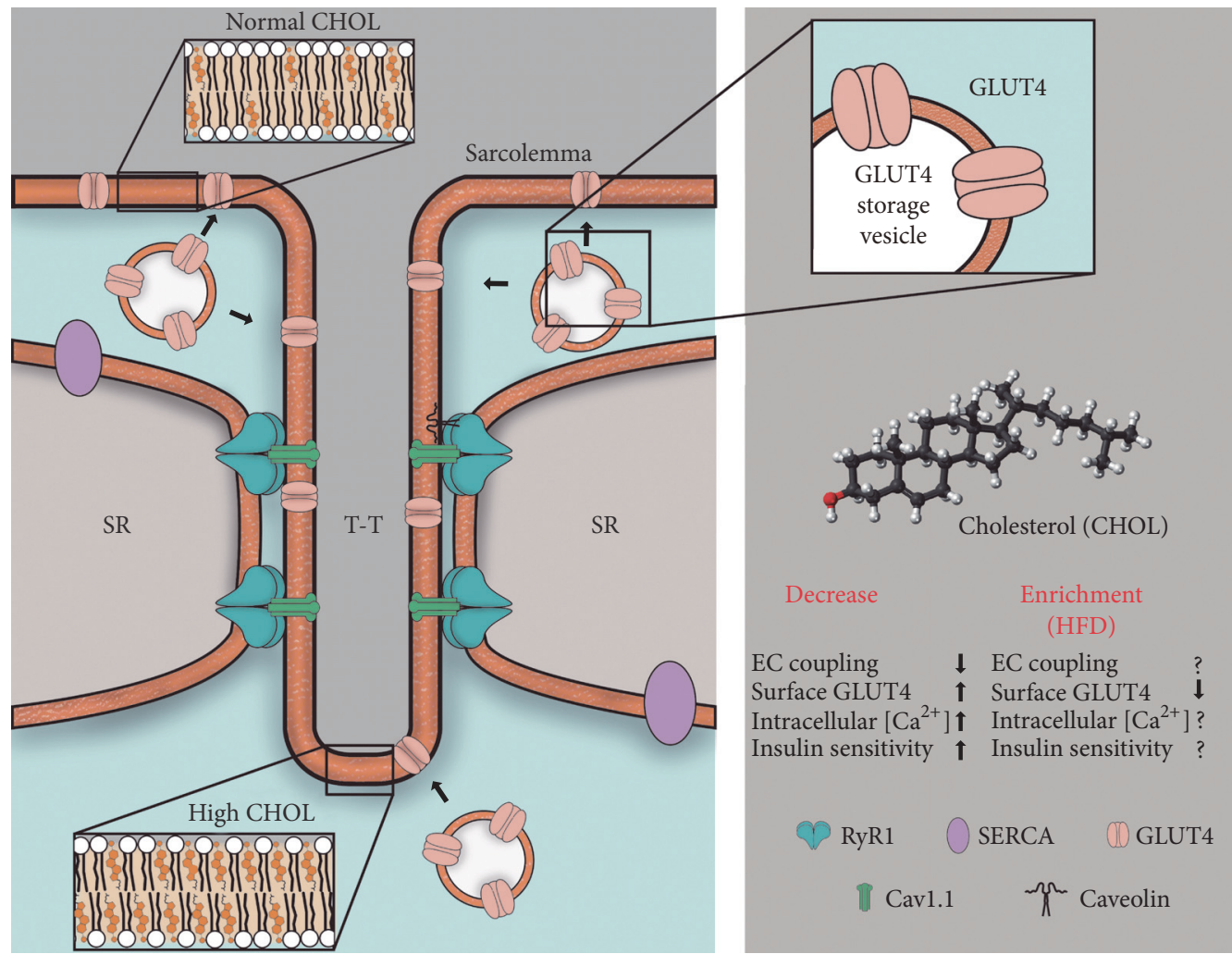

Figure 1: Schematic figure showing the T-tubule (T-T) system and its high cholesterol content compared with the surface membrane (sarcolemma, see inserts). Note the main proteins of the EC coupling complex and the sites of GLUT4 translocation. In response to insulin, the GLUT4 transporters translocate mainly to the T-tubule system, where EC coupling takes place, and also to the surface membrane region. Modifications of cholesterol content affect both EC coupling and GLUT4 mediated glucose transport in skeletal muscle. $\uparrow:$ increment; $\downarrow$ : decrease; ?: unknown effect.

potential exhibited by polyunsaturated membranes [42]. Currently, there is no information to our knowledge regarding the effects of obesity, metabolic syndrome, or cholesterol lowering treatments on dipole potential.

The manipulation of membrane cholesterol content is useful to study its effects on cellular physiology. The most common and simple approach has been to treat membranes with cyclodextrins, a family of cyclic compounds, which have a central hydrophobic pocket that extracts cholesterol from the cell membranes [43]. To date, however, only few studies, some of which are presented below, have provided information on how cholesterol modulation affects skeletal muscle protein functionality.

\section{T-Tubule Structure and Composition}

The T-tubule system of skeletal muscle is an intricate network composed of narrow tubules of around $40-85 \mathrm{~nm}$ in diameter which originate from deep invaginations of the surface plasma membrane [44]. This membrane system represents around $80 \%$ of the total plasma membrane surface of skeletal muscle [45]. Early studies using differential centrifugation reported that mammalian T-tubule membranes have a high proportion of cholesterol and sphingolipids [9] which resemble the composition of cholesterol-enriched lipid rafts domains [11]. Moreover, electron paramagnetic resonance assays indicate that at physiological temperature the lipid phase of T-tubule membranes is remarkably less fluid than that of other mammalian plasma membranes [10], resembling the low fluidity of thermophilic bacterial membranes. The T-tubules contain many proteins involved in EC coupling and other signaling processes; membrane cholesterol levels modulate the function of several of these proteins, including Cavl.1 [46], caveolin-3 [47], and $\mathrm{Na}^{+} / \mathrm{K}^{+}$- ATPase [48].

\section{T-Tubule Cholesterol Levels Influence the EC Coupling Process}

During muscle contraction, the action potential elicited at the neuromuscular junction propagates through the surface membrane into the T-tubule network, which is a key element in the EC coupling process [7]. The T-tubule membrane is flanked by two junctional sarcoplasmic reticulum (SR) membranes, forming structures known as triads which allow the direct interaction of the T-tubule residing protein Cavl.1 with Ryanodine receptor type 1 (RyR1), an integral SR membrane protein [7]. During EC coupling, Cavl.1 works as voltage sensor and commands transient RyR1 opening in response to membrane depolarization; the subsequent $\mathrm{Ca}^{2+}$ release from the SR produces muscle contraction [6]. 
It has been proposed that membrane cholesterol can modulate Cav1.1 activity [46]. In mechanically skinned fibers, in which the surface membrane is removed leaving the Tsystem intact, cholesterol depletion with $\mathrm{M} \beta \mathrm{CD}$ induces $\mathrm{T}$ tubule system depolarization without changes in its integrity [49]. Moreover, cholesterol depletion from intact fetal skeletal muscle using $\mathrm{M} \beta \mathrm{CD}$ decreases Cav1.1 $\mathrm{Ca}^{2+}$ currents and shifts their voltage dependence to more positive values; it is important to remark that $\mathrm{M} \beta \mathrm{CD}$ saturated with cholesterol does not affect Cavl.1 function [46].

We have shown that partial cholesterol removal from dissociated adult fibers inhibits EC coupling and depolarizes the fibers [11]. Cholesterol removal with $\mathrm{M} \beta \mathrm{CD}$ also increases the resting $\mathrm{Ca}^{2+}$ level, apparently by stimulating $\mathrm{Ca}^{2+}$ release from internal stores [11], suggesting that plasma membrane cholesterol might modulate functional interactions of the Ttubule membranes with the intracellular $\mathrm{Ca}^{2+}$ stores.

Caveolin-3 is a cholesterol-binding protein [47], which directly interacts with Cav1.1 [50] and modulates its $\mathrm{Ca}^{2+}$ channel function [51]. A recent report showed that cholesterol also modulates cardiac EC coupling and contraction [52]. Collectively, these results raise the possibility that cholesterol modulates striated muscle EC coupling by direct interaction with the protein complex engaged in this process and indirectly by modulating the lipid environment and accessory proteins such as caveolin-3.

\section{Cholesterol: A Novel Regulator in GLUT4 Translocation}

Skeletal muscle is a major contributor to whole-body metabolism; it is the largest insulin-sensitive tissue in the body, which makes it a key locus for insulin-stimulated glucose uptake [53]. In humans under euglycemic, hyperinsulinemic conditions, around $80 \%$ of body glucose uptake occurs in skeletal muscle, which represents a central component of glucose homeostasis [12]. In addition, this tissue is also an important consumer of fatty acids, which together with glucose constitute the principal energy sources of skeletal muscle [54].

The glucose transporter GLUT4 is one of fourteen members of the glucose transport family and displays high affinity for glucose [55]. Patients with IR and type 2 diabetes mellitus (T2DM) show defects in insulin-stimulated glucose metabolism in skeletal muscle. These alterations have been attributed to a disturbance in glucose transport, resulting mainly from dysregulated GLUT4 trafficking, the predominant insulin-sensitive glucose transporter expressed in skeletal muscle [56-58]. Both insulin and muscle contraction induce GLUT4 translocation to the skeletal muscle plasma membrane [59], presumably by engaging separate signaling pathways. The increase of surface GLUT4 occurs as a result of translocation of GLUT4-containing intracellular vesicles to the plasma membrane [60].

In order to enter into the muscle cell, glucose delivered by blood flow must be transported across the surface membrane and the T-tubule membranes into the cytoplasm, where it is trapped by hexokinase II action [58]. Insulin binding to the insulin receptor (InsR) promotes a conformational change in the receptor which leads to the transphosphorylation in tyrosine residues of its cytoplasmic $\beta$ subunits [61]. The activated InsR phosphorylates, among other proteins, the main InsR substrate (IRS) proteins, including IRS-1 and IRS-2. Tyrosinephosphorylated IRS-1 and IRS-2 serve as docking sites for SH2 domain-containing proteins, such as class IA (p85/p110type) phosphatidylinositol 3 kinase (PI3K) [62]. The activation of this lipid kinase increases phosphatidylinositol3,4,5-trisphosphate (PIP3) levels at the inner face of the plasma membrane and recruits pleckstrin $(\mathrm{PH})$ homology domain-containing proteins, which are essential for insulinstimulated GLUT4 translocation and the ensuing glucose uptake [8]. The $\mathrm{PH}$ domain-containing Akt protein is a cytoplasmic serine-threonine kinase that plays a fundamental role in mediating insulin-stimulated GLUT4 translocation; Akt organizes several downstream molecules that involve successive steps, including AS-160 activation and Rab Family GTPases, which finally position GLUT4 in the plasma membrane and promote GLUT4-mediated glucose transport [13, 63]. The increase in plasma membrane GLUT4 occurs due to a large increase in the rate of GLUT4 exocytosis, coupled with a smaller decrease in the rate of GLUT4 endocytosis [60]. The continuous recycling of GLUT4 offers the flexibility to regulate both its exocytic and endocytic rates. In cultured adipose and muscle cells, insulin rapidly stimulates the rate of exocytosis of GLUT4 transporters [64]. However, there is limited information about the mechanisms that regulate GLUT4 endocytosis. Although insulin reduces GLUT4 endocytosis in adipose cells [65], it does not affect the rate of GLUT4 internalization in rat cardiomyocytes [66] or in skeletal muscle cell lines [67]. In adult skeletal muscle, GLUT4 accumulates in several intracellular compartments, and although it locates preferentially in perinuclear regions, it is also present in peripheral vesicles $[68,69]$. Functional studies in adipocytes and skeletal muscle cell lines indicate that insulin-derived cellular signals promote GLUT4 translocation to the plasma membrane from a specialized compartment termed GLUT4 storage vesicles [60].

Various proteins including actin, actin dynamics, and microtubular motors intricately regulate the process of GLUT4 vesicle mobilization, tethering, docking, and fusion in response to insulin. GLUT4 is internalized via clathrinmediated endocytosis or via cholesterol-dependent but clathrin-independent endocytosis [60]. However, there are few studies addressing the role of membrane cholesterol in GLUT4 traffic.

In adipocyte and skeletal muscle cell under basal conditions, around 5\% of GLUT4 is present in the surface membranes; insulin stimulation increases its level to about $50 \%$ [70]. Early studies by nuclear magnetic resonance $[71,72]$ complemented with more recent reports have shown that GLUT4 translocation is defective in T2DM patients $[8,73]$. The majority of GLUT4-containing vesicles do not move long distances but are depleted locally in the surface membrane or T-tubule regions [8,74]. Moreover, analysis of GLUT4 translocation in insulin-resistant muscle showed that GLUT4 recruitment is affected primarily in the T-tubule region [8]. Muscles subjected to osmotic shock to dissociate the T-tubule connection with the surface membrane have their T-tubule 
network with no access to insulin and glucose from the extracellular fluid $[8,75]$. The dissociation of the T-tubule system reduces basal glucose transport by $50 \%$ and completely abolishes the insulin-induced increase in glucose transport [8], highlighting the critical role of the T-tubule system in insulin-mediated glucose transport [75]. Nevertheless, it is not clear why the dissociation of the T-tubule system inhibits insulin-dependent glucose transport through the surface membrane.

The mammalian T-tubule membranes are highly enriched in cholesterol and sphingolipids [9] endowing them with a rigid lipid environment with highly restricted membrane fluidity properties [10]. GLUT4 translocation occurs at cholesterol-rich microdomains [76], suggesting that changes in cholesterol levels modulate insulin-stimulated GLUT4 exocytosis. In fact, current evidence supports the hypothesis that increased plasma membrane cholesterol levels have a key role in the impaired GLUT4 traffic observed in IR and T2DM, since glucose-intolerant animal models and humans accumulate cholesterol in their skeletal muscle membranes [14]. Recently, using HFD-fed animals as a model of IR, we reported that triad-enriched fractions isolated from the skeletal muscle of these obese animals have around 30\% higher cholesterol content than triads from lean control animals [15]. In addition, muscle fibers isolated from HFD-fed obese mice show a $40 \%$ decrease in insulin-stimulated glucose uptake rates compared to fibers from lean control mice. In HFD-fed mice, four subcutaneous injections of $\mathrm{M} \beta \mathrm{CD}$ improved their defective glucose tolerance test, normalized their high fasting glucose levels, and restored insulin-stimulated glucose uptake in adult skeletal muscle fibers [15]. In addition, preincubation of isolated muscle fibers with relatively low concentrations of $\mathrm{M} \beta \mathrm{CD}$ increases both basal glucose uptake and insulininduced glucose uptake in fibers from controls or HFD-fed mice. In muscle fibers from HFD-fed mice, $M \beta C D$ improves insulin sensitivity and Indinavir, a GLUT4 antagonist, prevents the stimulatory effects of $\mathrm{M} \beta \mathrm{CD}$ on glucose uptake [15]. In addition, $\mathrm{M} \beta \mathrm{CD}$ increases membrane GLUT4 content and elicits intracellular calcium signals that are inhibited by Dantrolene, an agent which blocks the functional interaction of Cavl.1 and RyR1 [77] and reduces $\mathrm{M} \beta \mathrm{CD}$-mediated glucose uptake [15]. Interestingly, L6 myotubes cultured in a hyperinsulinemic medium resembling in vivo conditions that promote the progression of insulin resistance display an increase in membrane cholesterol [78]. The increased cholesterol levels in the plasma membrane of L6 myotubes cells may impair insulin action through a loss of cortical filamentous actin (F-actin), leading to defective GLUT4 regulation by insulin and an increase in the hexosamine biosynthesis pathway [14].

Treatment with chromium picolinate, a compound that removes membrane cholesterol, activates GLUT4 trafficking and enhances insulin-stimulated glucose transport via a cholesterol-dependent mechanism [79]. In addition, chromium supplementation significantly improves fasting glycemia in T2DM patients [80]. Treatment with $\mathrm{M} \beta \mathrm{CD}$ reversibly decreases the cholesterol content of membranes in a dosedependent manner, leading to increased GLUT4 incorporation into the plasma membrane of L6 myotubes [14]. More recently, it has been reported that AMP-activated protein kinase (AMPK) enhances insulin-stimulated GLUT4 regulation via lowering membrane cholesterol levels [78]. All together, these reports suggest a novel aspect of GLUT4 regulation by cholesterol in skeletal muscle.

\section{Concluding Remarks and Perspectives}

The cholesterol content of the T-tubule membrane in skeletal muscle is significantly higher than the levels present in the plasma membrane of most cells. A decrease in membrane cholesterol with cholesterol-removing agents alters muscle function, affecting both the excitation-contraction coupling process and glucose transport mediated by GLUT4 translocation to the T-tubule membrane (Figure 1). In conditions such as high-fat diet-induced obesity, the cholesterol content of T-tubule membranes increases even further, making it likely that pathological conditions, such as insulin resistance and type 2 diabetes, entail increased T-tubule cholesterol content. Accordingly, restoring membrane cholesterol levels in the T-tubule system via increasing surface GLUT4 levels in response to insulin may constitute an interesting therapeutic target to ameliorate insulin resistance.

\section{Competing Interests}

The authors declare that there are no competing interests regarding the publication of this review article.

\section{Acknowledgments}

This work was supported by FONDECYT 11150243 (CONICYT-Chile) and FIOUCH-ENLACE 001/2015 (Facultad de Odontología, Universidad de Chile).

\section{References}

[1] I. Tabas, Biochemistry of Lipids, Lipoproteins and Membranes, Elsevier, 2008.

[2] P. L. Yeagle, "Cholesterol and the cell membrane," $B B A-$ Reviews on Biomembranes, vol. 822, no. 3-4, pp. 267-287, 1985.

[3] P. L. Yeagle, "Modulation of membrane function by cholesterol," Biochimie, vol. 73, no. 10, pp. 1303-1310, 1991.

[4] G. Van Meer, D. R. Voelker, and G. W. Feigenson, "Membrane lipids: where they are and how they behave," Nature Reviews Molecular Cell Biology, vol. 9, no. 2, pp. 112-124, 2008.

[5] C. J. Fielding and P. E. Fielding, "Membrane cholesterol and the regulation of signal transduction," Biochemical Society Transactions, vol. 32, no. 1, pp. 65-69, 2004.

[6] R. T. Rebbeck, Y. Karunasekara, P. G. Board, N. A. Beard, M. G. Casarotto, and A. F. Dulhunty, "Skeletal muscle excitationcontraction coupling: who are the dancing partners?" International Journal of Biochemistry and Cell Biology, vol. 48, no. 1, pp. 28-38, 2014.

[7] A. F. Dulhunty, "Excitation-contraction coupling from the 1950s into the new millennium," Clinical and Experimental Pharmacology and Physiology, vol. 33, no. 9, pp. 763-772, 2006.

[8] M. P. Czech and S. Corvera, "Signaling mechanisms that regulate glucose transport," Journal of Biological Chemistry, vol. 274, no. 4, pp. 1865-1868, 1999. 
[9] M. Rosemblatt, C. Hidalgo, C. Vergara, and N. Ikemoto, "Immunological and biochemical properties of transverse tubule membranes isolated from rabbit skeletal muscle," Journal of Biological Chemistry, vol. 256, no. 15, pp. 8140-8148, 1981.

[10] C. Hidalgo, "Lipid phase of transverse tubule membranes from skeletal muscle. An electron paramagnetic resonance study," Biophysical Journal, vol. 47, no. 6, pp. 757-764, 1985.

[11] G. Barrientos, P. Llanos, J. Hidalgo et al., "Cholesterol removal from adult skeletal muscle impairs excitation-contraction coupling and aging reduces caveolin-3 and alters the expression of other triadic proteins," Frontiers in Physiology, vol. 6, article 105, 15 pages, 2015.

[12] D. Thiebaud, E. Jacot, R. A. DeFronzo, E. Maeder, E. Jequier, and J. P. Felber, "The effect of graded doses of insulin on total glucose uptake, glucose oxidation, and glucose storage in man," Diabetes, vol. 31, no. 11, pp. 957-963, 1982.

[13] A. Klip, Y. Sun, T. T. Chiu, and K. P. Foley, "Signal transduction meets vesicle traffic: the software and hardware of GLUT4 translocation," American Journal of Physiology-Cell Physiology, vol. 306, no. 10, pp. C879-C886, 2014.

[14] K. M. Habegger, B. A. Penque, W. Sealls et al., "Fat-induced membrane cholesterol accrual provokes cortical filamentous actin destabilisation and glucose transport dysfunction in skeletal muscle," Diabetologia, vol. 55, no. 2, pp. 457-467, 2012.

[15] P. Llanos, A. Ferrat-Contreras, T. Georgiev et al., "The cholesterol-lowering agent methyl- $\beta$-cyclodextrin promotes glucose uptake via GLUT4 in adult muscle fibers and reduces insulin resistance in obese mice," American Journal of PhysiologyEndocrinology and Metabolism, vol. 308, no. 4, pp. E294-E305, 2015.

[16] P. Bhonagiri, G. R. Pattar, K. M. Habegger, A. M. McCarthy, L. Tackett, and J. S. Elmendorf, "Evidence coupling increased hexosamine biosynthesis pathway activity to membrane cholesterol toxicity and cortical filamentous actin derangement contributing to cellular insulin resistance," Endocrinology, vol. 152, no. 9, pp. 3373-3384, 2011.

[17] J. Gallová, D. Uhríková, A. Islamov, A. Kuklin, and P. Balgavý, "Effect of cholesterol on the bilayer thickness in unilamellar extruded DLPC and DOPC liposomes: SANS contrast variation study," General Physiology and Biophysics, vol. 23, no. 1, pp. 113128, 2004.

[18] R. A. Cooper, "Influence of increased membrane cholesterol on membrane fluidity and cell function in human red blood cells," Journal of Supramolecular and Cellular Biochemistry, vol. 8, no. 4, pp. 413-430, 1978.

[19] T. H. Haines, "Do sterols reduce proton and sodium leaks through lipid bilayers?" Progress in Lipid Research, vol. 40, no. 4, pp. 299-324, 2001.

[20] G. H. Patterson, K. Hirschberg, R. S. Polishchuk, D. Gerlich, R. D. Phair, and J. Lippincott-Schwartz, "Transport through the golgi apparatus by rapid partitioning within a two-phase membrane system," Cell, vol. 133, no. 6, pp. 1055-1067, 2008.

[21] J. Fantini and F. J. Barrantes, "Sphingolipid/cholesterol regulation of neurotransmitter receptor conformation and function," Biochimica et Biophysica Acta (BBA)_Biomembranes, vol. 1788, no. 11, pp. 2345-2361, 2009.

[22] T. Róg, M. Pasenkiewicz-Gierula, I. Vattulainen, and M. Karttunen, "Ordering effects of cholesterol and its analogues," Biochimica et Biophysica Acta-Biomembranes, vol. 1788, no. 1, pp. 97-121, 2009.
[23] D. J. Recktenwald and H. M. McConnell, "Phase equilibria in binary mixtures of phosphatidylcholine and cholesterol," Biochemistry, vol. 20, no. 15, pp. 4505-4510, 1981.

[24] T. P. W. McMullen, R. N. A. H. Lewis, and R. N. McElhaney, "Cholesterol-phospholipid interactions, the liquid-ordered phase and lipid rafts in model and biological membranes," Current Opinion in Colloid and Interface Science, vol. 8, no. 6, pp. 459-468, 2004.

[25] J. R. Silvius, "Role of cholesterol in lipid raft formation: lessons from lipid model systems," Biochimica et Biophysica ActaBiomembranes, vol. 1610, no. 2, pp. 174-183, 2003.

[26] J. Huang and G. W. Feigenson, "A microscopic interaction model of maximum solubility of cholesterol in lipid bilayers," Biophysical Journal, vol. 76, no. 4, pp. 2142-2157, 1999.

[27] L. J. Pike, "Rafts defined: a report on the Keystone symposium on lipid rafts and cell function," Journal of Lipid Research, vol. 47, no. 7, pp. 1597-1598, 2006.

[28] K. Simons and E. Ikonen, "Functional rafts in cell membranes," Nature, vol. 387, no. 6633, pp. 569-572, 1997.

[29] H. An, M. R. Nussio, M. G. Huson, N. H. Voelcker, and J. G. Shapter, "Material properties of lipid microdomains: forcevolume imaging study of the effect of cholesterol on lipid microdomain rigidity," Biophysical Journal, vol. 99, no. 3, pp. 834-844, 2010.

[30] O. G. Mouritsen and M. Bloom, "Mattress model of lipidprotein interactions in membranes," Biophysical Journal, vol. 46, no. 2, pp. 141-153, 1984.

[31] H. Li and V. Papadopoulos, "Peripheral-type benzodiazepine receptor function in cholesterol transport. Identification of a putative cholesterol recognition/interaction amino acid sequence and consensus pattern," Endocrinology, vol. 139, no. 12, pp. 4991-4997, 1998.

[32] J. Fantini and F. J. Barrantes, "How cholesterol interacts with membrane proteins: an exploration of cholesterol-binding sites including CRAC, CARC, and tilted domains," Frontiers in Physiology, vol. 4, article no. 31, 2013.

[33] C. J. Baier, J. Fantini, and F. J. Barrantes, "Disclosure of cholesterol recognition motifs in transmembrane domains of the human nicotinic acetylcholine receptor," Scientific Reports, vol. 1, article 69, 2011.

[34] J. Fantini, C. Di Scala, L. S. Evans, P. T. F. Williamson, and F. J. Barrantes, "A mirror code for protein-cholesterol interactions in the two leaflets of biological membranes," Scientific Reports, vol. 6, article 21907, 2016.

[35] R. F. Flewelling and W. L. Hubbell, "The membrane dipole potential in a total membrane potential model. Applications to hydrophobic ion interactions with membranes," Biophysical Journal, vol. 49, no. 2, pp. 541-552, 1986.

[36] J. C. Franklin and D. S. Cafiso, "Internal electrostatic potentials in bilayers: measuring and controlling dipole potentials in lipid vesicles," Biophysical Journal, vol. 65, no. 1, pp. 289-299, 1993.

[37] L. Wang, "Measurements and implications of the membrane dipole potential," Annual Review of Biochemistry, vol. 81, pp. 615-635, 2012.

[38] T. Starke-Peterkovic, N. Turner, P. L. Else, and R. J. Clarke, "Electric field strength of membrane lipids from vertebrate species: membrane lipid composition and $\mathrm{Na}^{+}-\mathrm{K}^{+}$-ATPase molecular activity," American Journal of Physiology-Regulatory, Integrative and Comparative Physiology, vol. 288, pp. R663-R670, 2005.

[39] B. Maggio, "Modulation of phospholipase A2 by electrostatic fields and dipole potential of glycosphingolipids in monolayers," Journal of Lipid Research, vol. 40, no. 5, pp. 930-939, 1999. 
[40] T. Starke-Peterkovic, N. Turner, M. F. Vitha, M. P. Waller, D. E. Hibbs, and R. J. Clarke, "Cholesterol effect on the dipole potential of lipid membranes," Biophysical Journal, vol. 90, no. 11, pp. 4060-4070, 2006.

[41] P. Singh, S. Haldar, and A. Chattopadhyay, "Differential effect of sterols on dipole potential in hippocampal membranes: implications for receptor function," Biochimica et Biophysica ActaBiomembranes, vol. 1828, no. 3, pp. 917-923, 2013.

[42] S. Haldar, R. K. Kanaparthi, A. Samanta, and A. Chattopadhyay, "Differential effect of cholesterol and its biosynthetic precursors on membrane dipole potential," Biophysical Journal, vol. 102, no. 7, pp. 1561-1569, 2012.

[43] R. Zidovetzki and I. Levitan, "Use of cyclodextrins to manipulate plasma membrane cholesterol content: evidence, misconceptions and control strategies," Biochimica et Biophysica Acta (BBA)-Biomembranes, vol. 1768, no. 6, pp. 1311-1324, 2007.

[44] W. Melzer, A. Herrmann-Frank, and H. C. Lüttgau, "The role of $\mathrm{Ca}^{2+}$ ions in excitation-contraction coupling of skeletal muscle fibres," BBA-Reviews on Biomembranes, vol. 1241, no. 1, pp. 59116, 1995.

[45] L. Al-Qusairi and J. Laporte, "T-tubule biogenesis and triad formation in skeletal muscle and implication in human diseases," Skeletal Muscle, vol. 1, no. 1, article no. 26, 2011.

[46] S. Pouvreau, C. Berthier, S. Blaineau, J. Amsellem, R. Coronado, and C. Strube, "Membrane cholesterol modulates dihydropyridine receptor function in mice fetal skeletal muscle cells," Journal of Physiology, vol. 555, no. 2, pp. 365-381, 2004.

[47] M. Murata, J. Peränen, R. Schreiner, F. Wieland, T. V. Kurzchalia, and K. Simons, "VIP21/caveolin is a cholesterol-binding protein," Proceedings of the National Academy of Sciences of the United States of America, vol. 92, no. 22, pp. 10339-10343, 1995.

[48] R. Vemuri and K. D. Philipson, "Influence of sterols and phospholipids on sarcolemmal and sarcoplasmic reticular cation transporters," The Journal of Biological Chemistry, vol. 264, no. 15 , pp. 8680-8685, 1989.

[49] B. S. Launikonis and D. George Stephenson, "Effects of membrane cholesterol manipulation on excitation-contraction coupling in skeletal muscle of the toad," Journal of Physiology, vol. 534, no. 1, pp. 71-85, 2001.

[50] H. Couchoux, H. Bichraoui, C. Chouabe et al., "Caveolin3 is a direct molecular partner of the Cavl.1 subunit of the skeletal muscle L-type calcium channel," International Journal of Biochemistry and Cell Biology, vol. 43, no. 5, pp. 713-720, 2011.

[51] H. Couchoux, B. Allard, C. Legrand, V. Jacquemond, and C. Berthier, "Loss of caveolin-3 induced by the dystrophy-associated P104L mutation impairs L-type calcium channel function in mouse skeletal muscle cells," Journal of Physiology, vol. 580, no. 3, pp. 745-754, 2007.

[52] M. Z. Haque, V. J. McIntosh, A. B. Abou Samra, R. M. Mohammad, and R. D. Lasley, "Cholesterol depletion alters cardiomyocyte subcellular signaling and increases contractility," PLoS ONE, vol. 11, no. 7, Article ID e0154151, 2016.

[53] C. S. Stump, E. J. Henriksen, Y. Wei, and J. R. Sowers, "The metabolic syndrome: role of skeletal muscle metabolism," Annals of Medicine, vol. 38, no. 6, pp. 389-402, 2006.

[54] E. H. Koh, W. J. Lee, M.-S. Kim, J.-Y. Park, I. K. Lee, and K.-U. Lee, "Intracellular fatty acid metabolism in skeletal muscle and insulin resistance," Current diabetes reviews, vol. 1, no. 3, pp. 331336, 2005.

[55] R. Augustin, "The protein family of glucose transport facilitators: it's not only about glucose after all," IUBMB Life, vol. 62, no. 5, pp. 315-333, 2010.
[56] G. L. Dohm, E. B. Tapscott, W. J. Pories et al., "An in vitro human muscle preparation suitable for metabolic studies. Decreased insulin stimulation of glucose transport in muscle from morbidly obese and diabetic subjects," Journal of Clinical Investigation, vol. 82, no. 2, pp. 486-494, 1988.

[57] J. W. Ryder, J. Yang, D. Galuska et al., "Use of a novel impermeable biotinylated photolabeling reagent to assess insulinand hypoxia-stimulated cell surface GLUT4 content in skeletal muscle from type 2 diabetic patients," Diabetes, vol. 49 , no. 4 , pp. 647-654, 2000.

[58] J. S. Bogan, "Regulation of glucose transporter translocation in health and diabetes," Annual Review of Biochemistry, vol. 81, pp. 507-532, 2012.

[59] H. P. M. M. Lauritzen, "Insulin- and contraction-induced glucose transporter 4 traffic in muscle: insights from a novel imaging approach," Exercise and Sport Sciences Reviews, vol. 41, no. 2, pp. 77-86, 2013.

[60] K. Foley, S. Boguslavsky, and A. Klip, "Endocytosis, recycling, and regulated exocytosis of glucose transporter 4," Biochemistry, vol. 50, no. 15, pp. 3048-3061, 2011.

[61] H. Zaid, C. N. Antonescu, V. K. Randhawa, and A. Klip, "Insulin action on glucose transporters through molecular switches, tracks and tethers," Biochemical Journal, vol. 413, no. 2, pp. 201$215,2008$.

[62] R. T. Watson and J. E. Pessin, "GLUT4 translocation: the last 200 nanometers," Cellular Signalling, vol. 19, no. 11, pp. 2209-2217, 2007.

[63] S. Ishikura, P. J. Bilan, and A. Klip, "Rabs 8A and 14 are targets of the insulin-regulated Rab-GAP AS160 regulating GLUT4 traffic in muscle cells," Biochemical and Biophysical Research Communications, vol. 353, no. 4, pp. 1074-1079, 2007.

[64] C. M. Taniguchi, B. Emanuelli, and C. R. Kahn, "Critical nodes in signalling pathways: insights into insulin action," Nature Reviews Molecular Cell Biology, vol. 7, no. 2, pp. 85-96, 2006.

[65] M. P. Czech and J. M. Buxton, "Insulin action on the internalization of the GLUT4 glucose transporter in isolated rat adipocytes," The Journal of Biological Chemistry, vol. 268, no. 13, pp. 9187-9190, 1993.

[66] J. Yang and G. D. Holman, "Insulin and contraction stimulate exocytosis, but increased AMP-activated protein kinase activity resulting from oxidative metabolism stress slows endocytosis of GLUT4 in cardiomyocytes," Journal of Biological Chemistry, vol. 280, no. 6, pp. 4070-4078, 2005.

[67] C. N. Antonescu, M. Díaz, G. Femia, J. V. Planas, and A. Klip, "Clathrin-dependent and independent endocytosis of glucose transporter 4 (GLUT4) in myoblasts: regulation by mitochondrial uncoupling," Traffic, vol. 9, no. 7, pp. 1173-1190, 2008.

[68] T. Ploug, B. Van Deurs, H. Ai, S. W. Cushman, and E. Ralston, "Analysis of GLUT4 distribution in whole skeletal muscle fibers: identification of distinct storage compartments that are recruited by insulin and muscle contractions," Journal of Cell Biology, vol. 142, no. 6, pp. 1429-1446, 1998.

[69] J. D. Schertzer, C. N. Antonescu, P. J. Bilan et al., "A transgenic mouse model to study glucose transporter 4 myc regulation in skeletal muscle," Endocrinology, vol. 150, no. 4, pp. 1935-1940, 2009.

[70] O. J. Martin, A. Lee, and T. E. McGraw, "GLUT4 distribution between the plasma membrane and the intracellular compartments is maintained by an insulin-modulated bipartite dynamic mechanism," Journal of Biological Chemistry, vol. 281, no. 1, pp. 484-490, 2006. 
[71] G. W. Cline, K. F. Petersen, M. Krssak et al., "Impaired glucose transport as a cause of decreased insulin-stimulated muscle glycogen synthesis in type 2 diabetes," New England Journal of Medicine, vol. 341, no. 4, pp. 240-246, 1999.

[72] G. I. Shulman, "Cellular mechanisms of insulin resistance," Journal of Clinical Investigation, vol. 106, no. 2, pp. 171-176, 2000.

[73] W. T. Garvey, L. Maianu, J.-H. Zhu, G. Brechtel-Hook, P. Wallace, and A. D. Baron, "Evidence for defects in the trafficking and translocation of GLUT4 glucose transporters in skeletal muscle as a cause of human insulin resistance," Journal of Clinical Investigation, vol. 101, no. 11, pp. 2377-2386, 1998.

[74] H. P. M. M. Lauritzen, H. Galbo, J. Brandauer, L. J. Goodyear, and T. Ploug, "Large GLUT4 vesicles are stationary while locally and reversibly depleted during transient insulin stimulation of skeletal muscle of living mice imaging analysis of GLUT4enhanced green fluorescent protein vesicle dynamics," Diabetes, vol. 57, no. 2, pp. 315-324, 2008.

[75] W. Wang, P. A. Hansen, B. A. Marshall, J. O. Holloszy, and M. Mueckler, "Insulin unmasks a COOH-terminal Glut4 epitope and increases glucose transport across T-tubules in skeletal muscle," Journal of Cell Biology, vol. 135, no. 2, pp. 415-430, 1996.

[76] K. Fecchi, D. Volonte, M. P. Hezel, K. Schmeck, and F. Galbiati, "Spatial and temporal regulation of GLUT4 translocation by flotillin-1 and caveolin-3 in skeletal muscle cells," FASEB Journal, vol. 20, no. 6, pp. 705-707, 2006.

[77] R. A. Bannister, I. N. Pessah, and K. G. Beam, “The skeletal Ltype $\mathrm{Ca}^{2+}$ current is a major contributor to excitation-coupled $\mathrm{Ca}^{2+}$ entry," Journal of General Physiology, vol. 133, no. 1, pp. 7991, 2009.

[78] K. M. Habegger, N. J. Hoffman, C. M. Ridenour, J. T. Brozinick, and J. S. Elmendorf, "AMPK enhances insulin-stimulated GLUT4 regulation via lowering membrane cholesterol," Endocrinology, vol. 153, no. 5, pp. 2130-2141, 2012.

[79] N. J. Hoffman, B. A. Penque, K. M. Habegger, W. Sealls, L. Tackett, and J. S. Elmendorf, "Chromium enhances insulin responsiveness via AMPK," Journal of Nutritional Biochemistry, vol. 25, no. 5, pp. 565-572, 2014.

[80] E. M. Balk, A. Tatsioni, A. H. Lichtenstein, J. Lau, and A. G. Pittas, "Effect of chromium supplementation on glucose metabolism and lipids: a systematic review of randomized controlled trials," Diabetes Care, vol. 30, no. 8, pp. 2154-2163, 2007. 


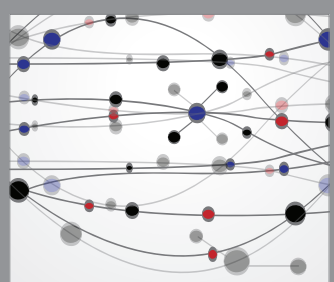

The Scientific World Journal
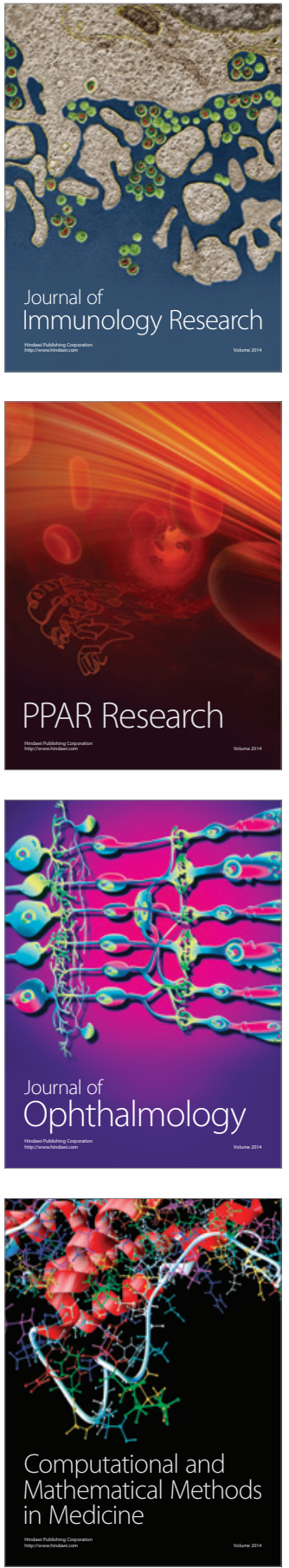

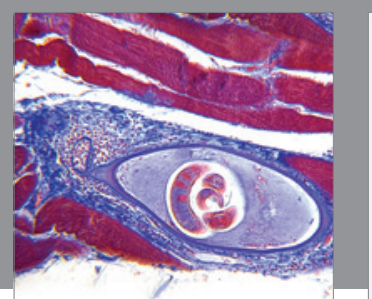

Gastroenterology Research and Practice
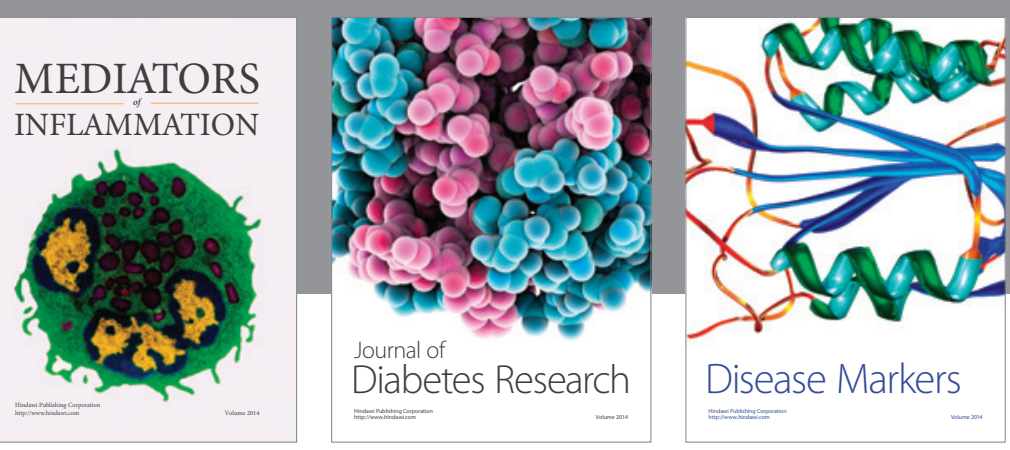

Disease Markers

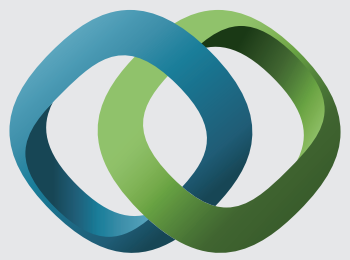

\section{Hindawi}

Submit your manuscripts at

https://www.hindawi.com
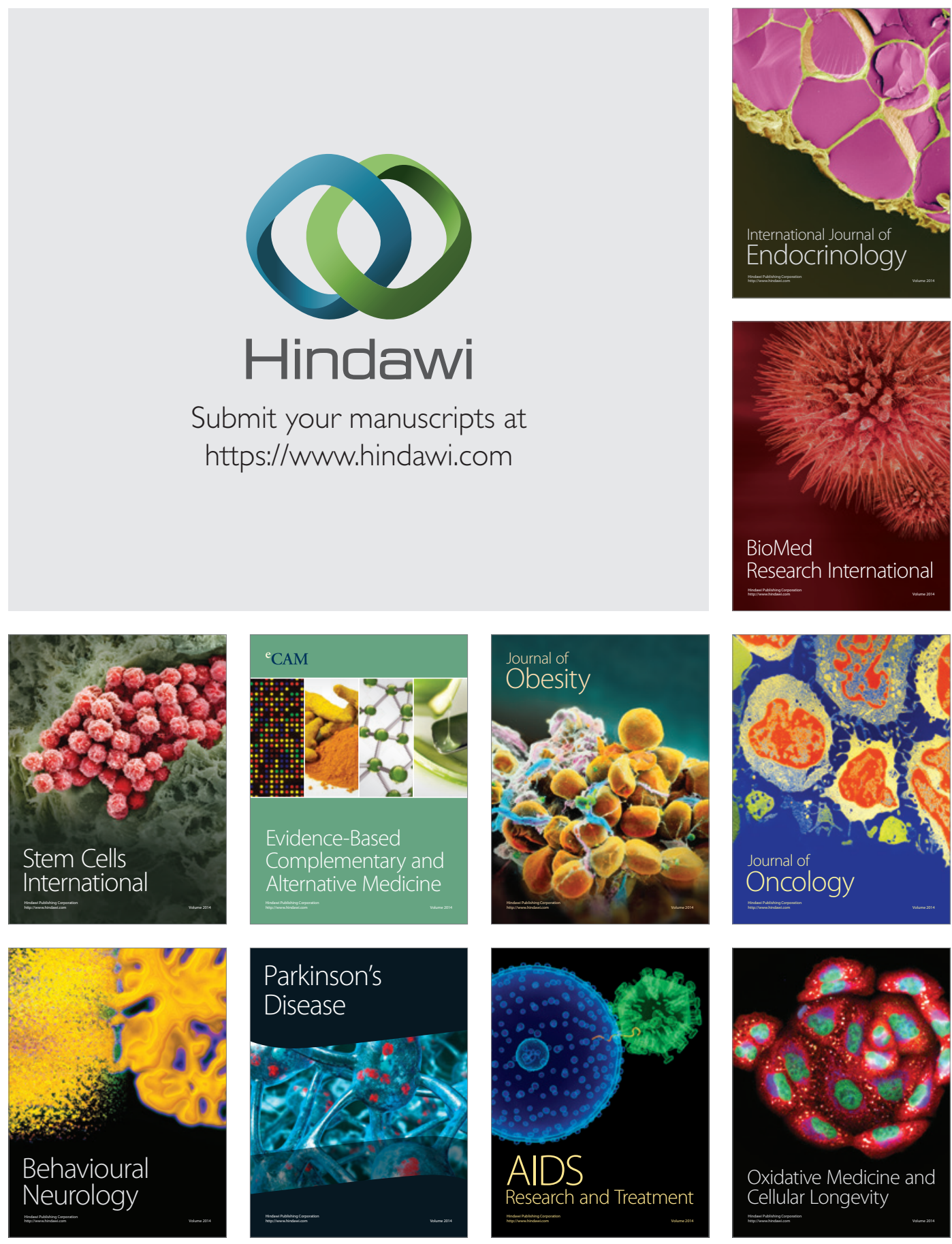